\title{
Can Platelet/Mean Platelet Volume ratio, Neutrophil/lymphocyte ratio, and Procalcitonin used for Predicting Prognosis in Acute Coronary Syndrome?
}

\author{
Sedat Özbay', Abuzer Coskun ${ }^{1 *}$, Sevki Hakan Eren² \\ 'Department of Emergency, Sivas Numune Hospital, Sivas, Turkey \\ ${ }^{2}$ Department of Emergency Medicine, Gaziantep University, Gaziantep, Turkey
}

\section{Article Info}

\section{Article Notes}

Received: January 11, 2020

Accepted: March 02, 2020

\section{*Correspondence:}

*Dr. Abuzer Coskun, Department of Emergency, Sivas Numune Hospital, Sivas, Turkey; Telephone: + 9044444 58; Fax: + 90 34622395 30; Email: dr.acoskun44@hotmail.com.

${ }^{\circ} 2020$ Coskun A. This article is distributed under the terms of the Creative Commons Attribution 4.0 International License.

\section{Keywords:}

Acute coronary syndrome

Mortality

Procalcitonin

Platelet/Mean platelet volume ratio

Neutrophil/Lymphocyte ratio
Abstract

Objective: This study aimed to evaluate acute coronary syndrome (ACS), serum procalcitonin levels, Platelet/Mean Platelet Volume ratio (PMR) and Neutrophil/lymphocyte ratio (NLR) concerning post-myocardial infarction (MI) complications, mortality, and morbidity.

Material and Method: The study included a total of 913 patients with ACS who presented to the emergency department with chest pain between January 2013 and December 2017. The patients were categorized as ST-elevated MI (STEMI), non-ST elevated MI (NSTEMI) and unstable angina (UA) according to the diagnosis. The demographic and laboratory characteristics of the patients were compared for three-vessel disease (TVD) and mortality rates.

Result: Post MI complications, TVD, and mortality were significant among acute coronary syndrome groups. The three-vessel disease was mostly observed in anterior MI with a maximum rate of $58(39.7 \%)$. Mortality was found as 23 $(16.3 \%)$ in anterior $\mathrm{MI}$ and $18(12.2 \%)$ in NSTEMI. Serum procalcitonin levels were highest in anterior MI. Platelet to Mean Platelet Volume ratio was higher in UA, whereas it was lower in STEMI and NSTEMI. Neutrophil to lymphocyte ratio was the lowest in UA. The cTn I values of STEMIs at $0,6,12$ hours were higher than UA, and the 12th-hour cTnl values in anterior MI were higher than NSTEMIs. It was found that procalcitonin and NLR had a positive correlation with post-MI complications, mortality, and TVD, whereas PMR exhibited a negative correlation.

Conclusion: The levels of procalcitonin, PMR, and NLR may be significant in respect of post-complications, mortality, and morbidity in acute coronary syndrome.

\section{Introduction}

Coronary artery disease remains the most common cause of mortality and morbidity worldwide. Despite recent advances in treatment options, increased life expectancy leads to an increase in the number of older and recurrent cardiovascular cases ${ }^{1}$. A coronary artery disease is a group of diseases whose incidence can be reduced when risk factors are managed properly.

Cellular events in platelets and white blood cells, endothelial and smooth muscle cells in the vessel wall and macrophages are important components of atherogenesis. Atherosclerotic plaque rupture or ulceration result in thrombus formation. Followingly, activation of platelets plays a very important role $\mathrm{e}^{2,3}$.

Mean Platelet Volume (MPV) is the index of platelet size that correlates with the functional status of platelets. Current studies demonstrate that MPV may be an indicator of recurrent myocardial 
infarction (MI) independent of established risk factors such as hypertension, dyslipidemia, increased fibrinogen and white blood cell count revealing high MPV levels in atherothrombosis ${ }^{4,5}$.

Since inflammation is a process that plays an important role in the initiation and progression of atherosclerosis, leukocytes are highly significant in this process. Neutrophils play a direct role in the initiation of plaque rupture ${ }^{6}$. It has been shown that neutrophil to lymphocyte ratio can be used as a marker for systemic inflammation in predicting morbidity, mortality, and risk of myocardial infarction in high-risk coronary artery disease ${ }^{7}$.

Troponin, together with tropomyosin, is a regulatory protein that plays a central role in the contraction and relaxation in striated cardiac and skeletal muscle tissues. Troponin, which is a complex of three regulatory proteins $\mathrm{T}$, I and C, provides the interaction of actin with myosin through calcium and is located in thin filaments. Troponins are sensitive and specific markers for cardiac muscle damage $^{8}$.

There have been many recent studies investigating cardiac markers. Procalcitonin is encoded by the CALC- 1 gene and it is produced from a 141 amino acid long preprocalcitonin. It binds to the endoplasmic reticulum of the $\mathrm{C}$-cells of the thyroid gland by the $\mathrm{N}$-terminus where it is cleaved by an endopeptidase ${ }^{9}$. Procalcitonin levels are found either very low or unquantifiable in healthy individuals ${ }^{10}$. Recent studies have reported that procalcitonin levels were correlated with the severity of atherosclerosis in patients with coronary artery disease, which was also linked to poor clinical outcomes ${ }^{11,12}$.

To the best of our knowledge, there have been no studies that investigated procalcitonin, PMR and NLR in acute coronary syndrome. In this study, we aimed to evaluate them in respect of post ACS complications, threevessel disease, and mortality.

\section{Material and method}

\section{Study design and population}

The data of a total of 913 patients (346 females, 567 males, mean age $63.84 \pm 12.18$ years; range 22 to 94 years) who presented to Sivas Numune Hospital Emergency Department with chest pain between January 2013 and December 2017 with the preliminary diagnosis of ACS were obtained retrospectively. Patients who were not measured for procalcitonin, biochemistry, and hemogram in the first 12 hours and patients who did not undergo postoperative angiography and echocardiography were excluded from the study. The study population included patients who were defined as ST-elevation MI (STEMI) [(inferior, posterior, anterior, lateral, high lateral, diffuse anterior MI)], nonSTelevation MI (NSTEMI) and high-risk unstable angina (UA).
The study consisted of patients who had chest pain and/or discomfort lasting at least 30 minutes and diagnosed with STEMI by electrocardiography according to the 2013 American College of Cardiology/American Heart Association guidelines ${ }^{13}$. UA/NSTEMI was defined according to the criteria of the 2014 American College of Cardiology/American Heart Association Guideline for the Management of Patients With NSTE-ACS. All patients underwent Transthoracic Echocardiography (TTE) for the presence of focal wall motion abnormalities. A Philips Epiq 7 Ultrasound Machine was used for TTE in this study.

Hemogram was measured using a Beckman Coulter Automated CBC Analyzer (Beckman Coulter, Inc., Fullerton, CA, USA). Demographic, clinical, and admission laboratory data of the patients presenting to the emergency department due to ACS, including the levels of procalcitonin and Tn I, were assessed by reviewing the hospital's medical records.

The study was conducted in compliance with the Declaration of Helsinki for Human Research and was approved by the institutional review board.

Written informed consent was obtained from all patients, and the study was approved by the Cumhuriyet University Medical School Ethical Committee.

Three-vessel disease (TVD); significant stenosis in the left anterior descending artery, circumflex artery, and right coronary arteries with at least $50 \%$ occlusion.

Cardiac Biomarker Analysis; Venous blood samples were obtained from the antecubital veins of the patients to measure serum levels of Troponin I by using the STAT Elecsys and Cobas e 411 Hitachi Roche analyzers. Troponin levels were measured at 0,6 , and 12 hours.

Procalcitonin was examined via the Mini Vidas brand device for 20-30 minutes. The values between 0 to $0.05 \mathrm{ng} /$ $\mathrm{ml}$ were accepted within the normal range, whereas the higher values were accepted as high.

Angiographic Analysis; All angiograms were evaluated by two experienced cardiologists who were blinded to the study. Discrepancies were resolved by consensus. The Gensini Score is a scale for coronary artery prevalence. Therefore it was used. PCT, TVD and prognosis were worse in patients with a score of more than 20 points. The extent and severity of coronary artery disease were assessed by the Gensini Score ${ }^{14}$.

\section{Statistical analysis}

The data obtained from the study were analyzed using SPSS 20 software package. Shapiro-Wilk-W test was used while investigating the significance of variables in distribution. When analyzing the differences between the groups, since the values of the variables are less than 0.05, the non-parametric test methods, Mann Whitney U and 
Kruskal Wallis-H Tests were used. The chi-square analysis was performed to examine the correlation between the groups of nominal variables. In cases where the expected values in the cells do not have sufficient volume in $2 \times 2$ tables, Fisher's Exact Test was used, and in RxC tables, association analysis was applied with the help of Monte Carlo Simulation (The expected values for each of the cells in the crosstab are not less than 5 (2x2-sized tables) or 2 (RxC-sized tables). Additionally, receiver operating characteristic (ROC) curve analysis was performed for TVD and mortality. When interpreting the results, 0.05 was used bilaterally. P values less than 0.05 were considered statistically significant.

\section{Result}

The clinical and demographic characteristics of the patients are listed in Table 1.

Gender, TVD, mortality, and complications were found to be significant between different groups of acute coronary syndrome. Both sexes had high levels of UA, whereas NSTEMI was more common in females and inferior MI was more common in males. The three-vessel disease was most frequently observed in anterior MI. Mortality was more frequent in STEMI (Table 2).

By gender; 49 (14.2\%) females and 131 (23.1\%) males had TVD. Mortality was determined as 18 (5.2\%) in females and $57(10.1 \%)$ in males.

The variables were not significant with gender age and NLR but were alive in terms of mortality and whether there was vascular occlusion in terms of death and TVD, the Mann-Whitney U test was significant (Table 3).

The Kruskal-Walles $\mathrm{H}$ test was statistically significant to ACS diagnoses of the variables (Table 4).

There was a significant correlation between procalcitonin, PMR and NLR (Table 5).

According to the ROC curve analysis, the optimal cutoff values of procalcitonin, PMR and NLR to determine the mortality positivity were found as AUC; $0.602: 0.347$ : 0.618,> 0.158:> 15.66:> 2.64, sensitivity 69.4\%: 88.9\%: $89.3 \%$ and specificity $58.7 \%$ : $81.3 \%: 71.7 \%$, respectively ( $\mathrm{p}=0.001$, Figure 1).

According to the ROC curve analysis, the optimal cutoff values of procalcitonin, PMR and NLR to determine the TVD positivity were found as AUC; 0.587: 0.309: 0.681,> 0.126:> 15.92:> 2.37, sensitivity $67.7 \%: 87.9 \%: 90.2 \%$ and specificity $58.9 \%$ : $76.1 \%: 70.8 \%$, respectively $(\mathrm{p}=0.001$, Figure 2).

\section{Discussion}

In this study, we aimed to investigate the correlation between the levels of PMR, NLR and procalcitonin and possible complications following ACS, TVD, and mortality among the emergency department admissions since there were a limited number of studies in this regard.

Coronary artery disease is a chronic inflammatory disorder initiated by atherosclerosis. Biasucci et al. ${ }^{15}$ suggested that procalcitonin was excreted depending on

Table 1: Baseline characteristics of study patients and laboratory finding

\begin{tabular}{|c|c|c|c|c|c|c|c|c|c|c|}
\hline \multicolumn{11}{|c|}{ Acute Coronary Syndrome } \\
\hline & All pat. & UA & IMI & PMI & AMI & LMI & CAMI & HLMI & NSTEMI & \\
\hline & n:913 & n:399 & $\mathrm{n}: 149$ & $\mathrm{n}: 8$ & $\mathrm{n}: 148$ & $\mathrm{n}: 12$ & $\mathrm{n}: 44$ & $\mathrm{n}: 12$ & n:141 & \\
\hline Baseline Characteristics & mean $\pm S D$ & mean $\pm S D$ & mean $\pm S D$ & mean $\pm S D$ & mean $\pm S D$ & mean $\pm S D$ & mean $\pm S D$ & mean $\pm S D$ & mean $\pm S D$ & p-value \\
\hline Age, $\mathrm{Yr}$ & $63.8 \pm 12.2$ & $61.7 \pm 12.8$ & $64.3 \pm 11.8$ & $70.4 \pm 11.9$ & $65.8 \pm 11.9$ & $69.3 \pm 1.0$ & $62.1 \pm 11.1$ & $58.4 \pm 5.1$ & $67.8 \pm 10.4$ & 0.001 \\
\hline Sex, Female/Male & $346 / 567$ & $184 / 215$ & $40 / 109$ & $2 / 6$ & $48 / 100$ & $3 / 9$ & $8 / 36$ & $5 / 7$ & $56 / 85$ & 0.001 \\
\hline \multicolumn{11}{|l|}{ Laboratory Finding } \\
\hline GS & $32.3 \pm 38.6$ & $13.9 \pm 25.4$ & $41.8 \pm 36.7$ & $39.7 \pm 69.2$ & $55.2 \pm 41.0$ & $63.5 \pm 45.8$ & $72.0 \pm 52.2$ & $41.5 \pm 34.2$ & $32.6 \pm 33.6$ & 0.001 \\
\hline Tn $1(0 \mathrm{~h})(\mathrm{ng} / \mathrm{mL})$ & $1.6 \pm 3.8$ & $0.0 \pm 0.0$ & $3.3 \pm 7.9$ & $2.1 \pm 2.1$ & $3.0 \pm 2.3$ & $2.4 \pm 1.6$ & $3.2 \pm 2.5$ & $3.3 \pm 3.6$ & $1.9 \pm 2.2$ & 0.001 \\
\hline $\operatorname{Tn} 2(6 \mathrm{~h})$ & $3.7 \pm 5.6$ & $0.1 \pm 0.2$ & $7.1 \pm 6.6$ & $5.4 \pm 6.8$ & $7.4 \pm 5,2$ & $6.2 \pm 4.2$ & $8.1 \pm 6.0$ & $6.7 \pm 4.9$ & $4.8 \pm 6.2$ & 0.001 \\
\hline Tn $3(12$ h) & $8.6 \pm 13.2$ & $0.1 \pm 0.3$ & $17.1 \pm 18.0$ & $11.8 \pm 16.1$ & $17.1 \pm 11.6$ & $15.7 \pm 11.9$ & $18.7 \pm 12.3$ & $14.5 \pm 10.0$ & $10.0 \pm 13.2$ & 0.001 \\
\hline Glu (mg/dL) & $137.6 \pm 71.9$ & $13.3 \pm 75.1$ & $138.6 \pm 76.0$ & $175.0 \pm 96.4$ & $139.4 \pm 66.7$ & $137.7 \pm 36.6$ & $142.9 \pm 63.4$ & $201.1 \pm 113.1$ & $137.9 \pm 59.9$ & 0.065 \\
\hline PLT (10^3/uL) & $232.4 \pm 68.1$ & $288.8 \pm 58.0$ & $191.6 \pm 35.5$ & $192.4 \pm 21.7$ & $187.9 \pm 35.9$ & $189.0 \pm 23.2$ & $185.0 \pm 30.1$ & $177.5 \pm 19.5$ & $188.3 \pm 37.6$ & 0.001 \\
\hline Neu (\%) & $5.7 \pm 3.8$ & $2.6 \pm 1.2$ & $8.0 \pm 3.1$ & $8.1 \pm 4.5$ & $8.2 \pm 3.2$ & $10.7 \pm 3.7$ & $8.6 \pm 4.2$ & $6.8 \pm 2.8$ & $7.8 \pm 3.2$ & 0.001 \\
\hline Lymph (\%) & $1.5 \pm 0.8$ & $1.1 \pm 0.6$ & $1.8 \pm 0.8$ & $1.9 \pm 1.4$ & $1.8 \pm 0.8$ & $2.0 \pm 1.0$ & $1.7 \pm 1.0$ & $1.6 \pm 0.9$ & $1.8 \pm 0.9$ & 0.001 \\
\hline PCT (ng/dL) & $0.3 \pm 0.4$ & $0.0 \pm 0.0$ & $0.3 \pm 0.4$ & $0.3 \pm 0.5$ & $0.9 \pm 0.3$ & $0.6 \pm 0.5$ & $0.8 \pm 0.4$ & $0.5 \pm 0.5$ & $0.4 \pm 0.3$ & 0.001 \\
\hline PMR & $29.6 \pm 12.5$ & $40.1 \pm 9.6$ & $21.9 \pm 7.6$ & $22.9 \pm 5.7$ & $21.7 \pm 7.5$ & $20.6 \pm 6.5$ & $20.6 \pm 6.2$ & $17.6 \pm 2.0$ & $21.3 \pm 7.6$ & 0.001 \\
\hline NLR & $4.3 \pm 3.3$ & $2.6 \pm 1.3$ & $5.4 \pm 4.3$ & $6.7 \pm 5.0$ & $5.6 \pm 3.6$ & $6.4 \pm 4.0$ & $5.8 \pm 3.1$ & $6.5 \pm 6.4$ & $5.3 \pm 3.5$ & 0.001 \\
\hline
\end{tabular}

Yr: year, SD: standard deviation, Pat: patients, UA: Unstable Angina, IMI: Inferior myocardial infarction, PMI: Posterior myocardial infarction, AMI: Anterior myocardial infarction, LMI: Lateral myocardial infarction, CAMI: Common anterior myocardial infarction, HLMI: High Lateral myocardial infarction, NSTEMI: Non-ST elevated MI, GS: Gensini Score, Glu: Glucose, LVEF: Left ventricular ejection fraction, Tn: Cardiac troponin, h: hour, PLT: Platelets, MPV: Mean Platelet Volume, Neu: Neutrophils, Lymph: Lymphocytes, PCT: Procalcitonin, PMR: Platelets/Mean Platelet Volume Rate, NLR: Neutrophils/ Lymphocytes Rate (The $\mathrm{p}$-value value was taken from the Kruskal-Wallis $\mathrm{H}$ test.) 
Table 2: Outcome Relationship Between Acute Coronary Syndrome and Variables

\begin{tabular}{|c|c|c|c|c|c|c|c|c|c|c|c|}
\hline \multicolumn{12}{|c|}{ Acute Coronary Syndrome } \\
\hline & & UA & IMI & PMI & AMI & LMI & CAMI & HLMI & NSTEMI & & \\
\hline & & $n(\%)$ & $n(\%)$ & $n(\%)$ & $n(\%)$ & $n(\%)$ & $n(\%)$ & $n(\%)$ & $n(\%)$ & $x^{2}$ & p-value \\
\hline \multirow{3}{*}{ Gender } & Female & $184(46.1)$ & $40(26.8)$ & $2(25.0)$ & $48(32.4)$ & $3(25.0)$ & $8(18.2)$ & $5(41.7)$ & $56(39.7)$ & \multirow{3}{*}{30.01} & \multirow{3}{*}{0.001} \\
\hline & Male & $215(53.9)$ & $109(73.2)$ & $6(75.0)$ & $100(67.6)$ & $9(75.0)$ & $36(81.8)$ & $7(58.3)$ & $85(60.3)$ & & \\
\hline & Total & $399(100)$ & 149(100) & $8(100)$ & $148(100)$ & $12(100)$ & $44(100)$ & $12(100)$ & $141(100)$ & & \\
\hline & & & & & & & & & & & \\
\hline \multirow{3}{*}{ TVD } & No & $376(94.2)$ & 109(73.2) & $6(75.0)$ & $90(60.8)$ & $5(41.7)$ & $23(52.3)$ & $10(83.3)$ & $114(80.9)$ & \multirow{3}{*}{122.66} & \multirow{3}{*}{0.001} \\
\hline & Yes & $23(5.8)$ & $40(26.8)$ & $2(25.0)$ & $58(39.2)$ & $7(58.3)$ & $21(47.7)$ & $2(16.7)$ & $27(19.1)$ & & \\
\hline & Total & $399(100)$ & $149(100)$ & $8(100)$ & $148(100)$ & $12(100)$ & $44(100)$ & $12(100)$ & $141(100)$ & & \\
\hline \multirow{8}{*}{ Complication } & No & $329(82.5)$ & $70(47.0)$ & $3(37.5)$ & $42(228.4)$ & $3(25.0)$ & $11(25.0)$ & $6(50.0)$ & $62(44.0)$ & \multirow{8}{*}{318.99} & \multirow{8}{*}{0.001} \\
\hline & IHF & $45(11.3)$ & $41(27.5)$ & $2(25.0)$ & $77(52.0)$ & $7(58.3)$ & $25(56,8)$ & $3(25.0)$ & $49(34.8)$ & & \\
\hline & VT & $6(1.5)$ & $4(2.7)$ & $1(12.5)$ & $13(8.8)$ & $0(0)$ & $5(11.4)$ & $1(8.3)$ & $15(10.6)$ & & \\
\hline & $A V B I$ & $5(1.3)$ & $23(15.4)$ & $2(25.0)$ & $1(0.7)$ & $1(8.3)$ & $0(0)$ & $1(8.3)$ & $1(0.7)$ & & \\
\hline & CT & $6(1.5)$ & $3(2.0)$ & $0(0)$ & $0(0)$ & $0(0)$ & $0(0)$ & $1(8.3)$ & $6(4.3)$ & & \\
\hline & APE & $8(2.0)$ & $8(5.4)$ & $0(0)$ & $15(10.1)$ & $1(8.3)$ & $3(6.8)$ & $1(8.3)$ & $7(5.0)$ & & \\
\hline & PI Eff & $0(0)$ & $0(0)$ & $0(0)$ & $0(0)$ & $0(0)$ & $0(0)$ & $0(0)$ & $1(0.7)$ & & \\
\hline & Total & $399(100)$ & $149(100)$ & $8(100)$ & $148(100)$ & $12(100)$ & $44(100)$ & $12(100)$ & $141(100)$ & & \\
\hline \multirow{3}{*}{ Mortality } & No & $389(97.5)$ & 134(89.9) & $7(87.5)$ & $130(87.8)$ & $11(91.7)$ & $39(88.6)$ & $10(8.3)$ & $118(83.7)$ & \multirow{3}{*}{35.15} & \multirow{3}{*}{0.001} \\
\hline & Yes & $10(2.5)$ & 15(10.1) & $1(12.5)$ & $18(12.2)$ & $1(8.3)$ & $5(11,4)$ & $2(16.7)$ & $23(16.3)$ & & \\
\hline & Total & $399(100)$ & 149(100) & $8(100)$ & $148(100)$ & $12(100)$ & $44(100)$ & $12(100)$ & $141(100)$ & & \\
\hline
\end{tabular}

TVD: Three-vessel disease, IHF: Ischemic Heart Failure, VT: Ventricular tachycardia, AV BI: Atrioventricular Blocs, CT: Cardiac Tamponade, APE: Acute Pulmonary Edema, PI Eff: Pleural effusion

Table 3: Analysis of variables in acute coronary syndrome by results

\begin{tabular}{|c|c|c|c|c|c|c|}
\hline \multicolumn{7}{|c|}{ Acute Coronary Syndrome } \\
\hline & \multicolumn{2}{|c|}{ Gender } & \multicolumn{2}{|c|}{ TVD } & \multicolumn{2}{|c|}{ Mortality } \\
\hline & \multicolumn{2}{|c|}{ n: 913} & \multicolumn{2}{|c|}{$\mathrm{n}: 180$} & \multicolumn{2}{|c|}{ n:75 } \\
\hline & $z$ & p-value & $z$ & p-value & $z$ & p-value \\
\hline AGE & -1.25 & 0.211 & -3.11 & 0.002 & -2.84 & 0.004 \\
\hline GS & -7.06 & 0.001 & -20.66 & 0.001 & -4.44 & 0.001 \\
\hline LVEF & -5.41 & 0.001 & -7.63 & 0.001 & -7.19 & 0.001 \\
\hline $\operatorname{Tn} 1(0 \mathrm{~h})$ & -3.56 & 0.001 & -9.92 & 0.001 & -6.40 & 0.001 \\
\hline $\operatorname{Tn} 2(6 \mathrm{~h})$ & -3.40 & 0.001 & -10.26 & 0.001 & -6.54 & 0.001 \\
\hline $\operatorname{Tn} 3$ (12 h) & -3.43 & 0.001 & -10.03 & 0.001 & -5.93 & 0.001 \\
\hline PLT & -2.60 & 0.009 & -7.85 & 0.001 & -4.17 & 0.001 \\
\hline MPV & -2.49 & 0.001 & -6.24 & 0.001 & -3.90 & 0.001 \\
\hline Neu & -3.22 & 0.001 & -8.29 & 0.001 & -4.36 & 0.001 \\
\hline Lymph & -3.00 & 0.003 & -4.36 & 0.001 & -3.27 & 0.001 \\
\hline РCT & -3.45 & 0.001 & -4.15 & 0.001 & -3.34 & 0.001 \\
\hline PMR & -2.78 & 0.005 & -7.93 & 0.001 & -4.38 & 0.001 \\
\hline NLR & -1.95 & 0.051 & -7.53 & 0.001 & -3.37 & 0.001 \\
\hline
\end{tabular}

TVD: Three-vessel disease, GS: Gensini Score, LVEF: Left ventricular ejection fraction, Tn: Cardiac troponin, h: hour, PLT: Platelets, MPV: Mean Platelet Volume, Neu: Neutrophils, Lymph: Lymphocytes, PCT: Procalcitonin, PMR: Platelets/Mean Platelet Volume Rate, NLR: Neutrophils/ Lymphocytes Rate

inflammatory events occurring during ACS. They noted that elevated procalcitonin levels were associated with poorer prognosis and correlated with the degree of atherosclerosis in coronary artery disease. In a similar study, Sinning et al. ${ }^{16}$ reported that initial elevated procalcitonin levels were associated with cardiovascular events and higher mortality during follow-up.

In our study, procalcitonin levels were similarly high in ACS. This elevation was found to be lower in UA,
Table 4: Analysis of the results of acute coronary syndrome by diagnosis

\begin{tabular}{|l|c|c|c|}
\hline \multicolumn{4}{|c|}{ Acute Coronary Syndrome } \\
\hline GS & mean \pm SD & H & p-value \\
\hline Glu & $32.26 \pm 38.63$ & 285.23 & 0.001 \\
\hline Tn 1 (0 h) & $137.64 \pm 71.95$ & 17.43 & 0.015 \\
\hline Tn 2 (6 h) & $1.57 \pm .77$ & 657.94 & 0.001 \\
\hline Tn 3 (12 h) & $3.74 \pm 5.58$ & 670.43 & 0.001 \\
\hline PCT & $8.58 \pm 13.19$ & 676.04 & 0.001 \\
\hline PMR & $0.30 \pm 0.41$ & 504.19 & 0.001 \\
\hline NLR & $29.60 \pm 12.49$ & 517.77 & 0.001 \\
\hline
\end{tabular}

GS: Gensini Score, Glu: Glucose, LVEF: Left ventricular ejection fraction, Tn: Cardiac troponin, h: hour, PCT: Procalcitonin, PMR: Platelets/Mean Platelet Volume Rate, NLR: Neutrophils/ Lymphocytes Rate

Table 5: Analysis of the relationship between procalcitonin, PMR, and NLR result form

\begin{tabular}{|l|c|c|c|c|c|c|}
\hline & \multicolumn{2}{|c|}{ Procalcitonin } & \multicolumn{2}{c|}{ PMR } & \multicolumn{2}{c|}{ NLR } \\
\hline & $\mathbf{r}$ & $\mathbf{p}$-value & $\mathbf{r}$ & $\mathbf{p}$-value & $\mathbf{r}$ & p-value \\
\hline GS & 0.284 & 0.001 & -0.391 & 0.001 & 0.398 & 0.001 \\
\hline LVEF & -0.240 & 0.001 & 0.403 & 0.001 & -0.378 & 0.001 \\
\hline BS & 0.043 & 0.196 & -0.029 & 0.383 & 0.057 & 0.084 \\
\hline Tn 1 (0 h) & 0.509 & 0.001 & -0.642 & 0.001 & 0.628 & 0.001 \\
\hline Tn 2 (6 h) & 0.513 & 0.001 & -0.643 & 0.001 & 0.623 & 0.001 \\
\hline Tn 3 (12 h) & 0.512 & 0.001 & -0.649 & 0.001 & 0.623 & 0.001 \\
\hline PCT & - & - & -0.460 & 0.001 & 0.436 & 0.001 \\
\hline PMR & -0.460 & 0.001 & - & - & -0.546 & 0.001 \\
\hline NLR & 0.436 & 0.001 & -0.546 & 0.001 & - & - \\
\hline
\end{tabular}

GS: Gensini Score, Glu: Glucose, Tn: Cardiac troponin, h: hour, PCT: Procalcitonin, PMR: Platelets/Mean Platelet Volume Rate, NLR: Neutrophils/ Lymphocytes Rate

which had low-level inflammation, compared to STEMI and NSTEMI. It was higher in anterior MI. Procalcitonin 


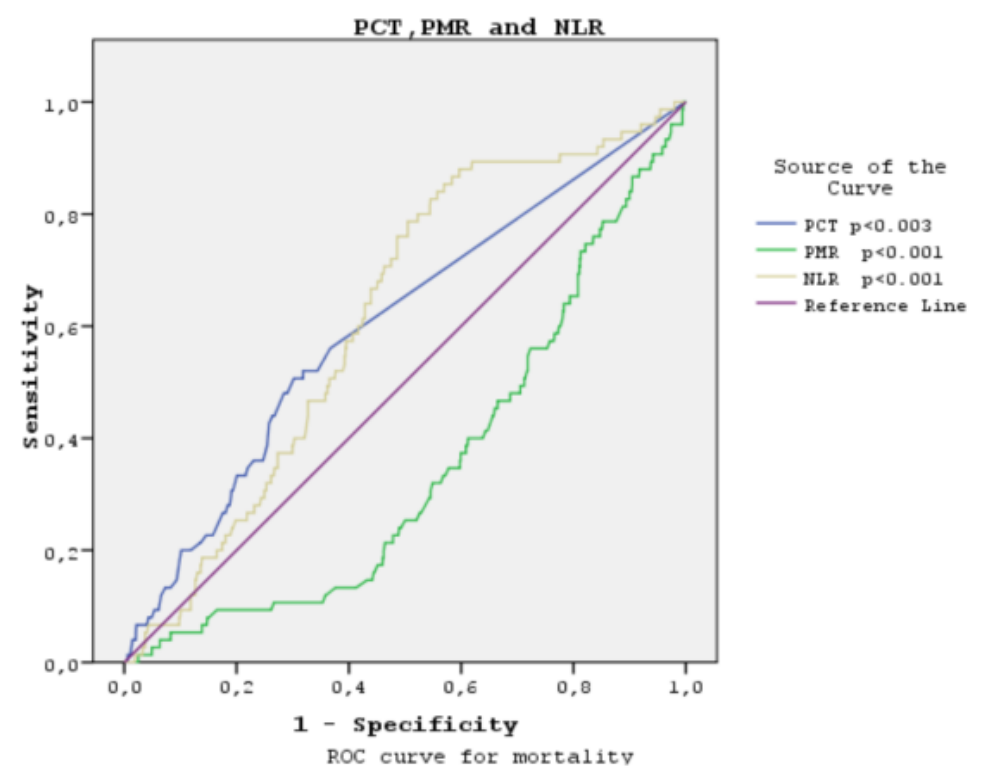

Figure 1: ROC curve analysis according to procalcitonin, PMR and NLR mortality positivity

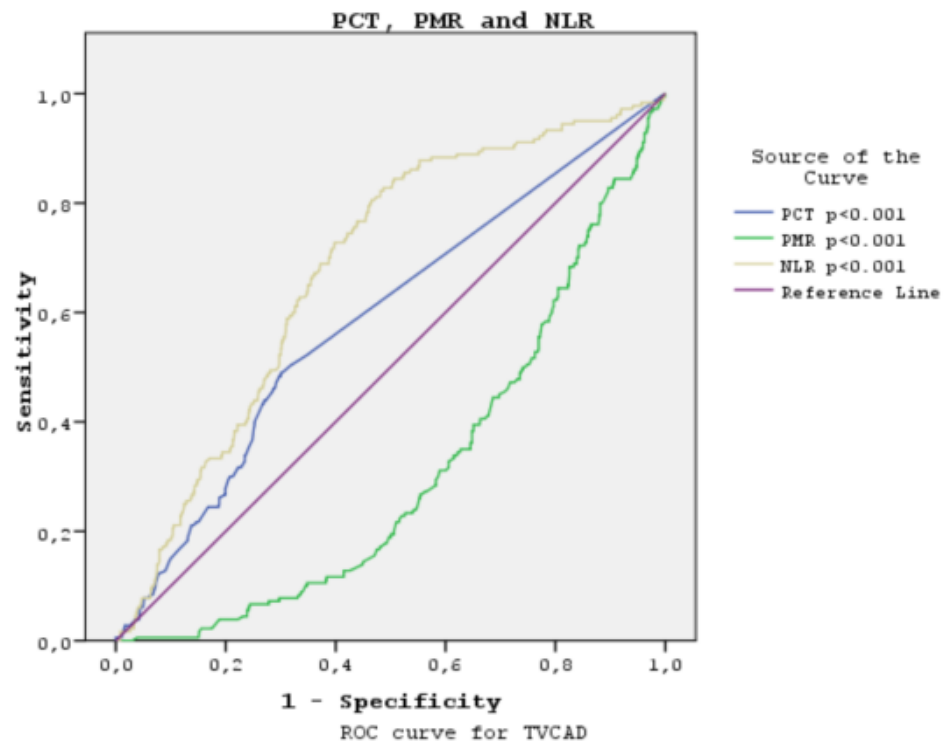

Figure 2: ROC curve analysis according to procalcitonin, PMR and NLR TVD positivity

levels were negatively correlated with PMR and positively correlated with NLR, which was consistent with the studies investigating post ACS complications.

In one of the studies investigating the correlation between troponin and mortality, Antman et al. ${ }^{17}$ reported that elevated Tn I in ACS patients was a good indicator for predicting the possible mortality. Kafkas et al. ${ }^{18}$ reported high levels of Tn I and procalcitonin in patients with ACS. Our study revealed that the levels of procalcitonin and Tn I levels at 0,6 , and $12 \mathrm{~h}$ had a strong positive correlation with mortality in ACS patients. The highest mortality was in anterior MI. Among all cases, mortality was 57 (10.1\%) in males and 18 (5.2\%) in females. Also, the sensitivity and specificity of procalcitonin for mortality were $69.4 \%$ and $58.7 \%$, respectively.

Senturk et al. ${ }^{19}$ demonstrated that the levels of troponin and procalcitonin were found to be higher in patients with TVD and also associated with mortality. Our study revealed high mortality, TVD, and complications in the groups with high levels of procalcitonin and Tn. Procalcitonin levels were high in anterior MI. The three-vessel disease was found to be high in anterior and inferior MI. Besides, STEMI 
had a higher gensini score and lower LVEF compared to the other groups. It was noted that high levels of procalcitonin, troponins, PMR and NLR were associated with mortality, TVD, complications and poor prognosis in acute coronary syndrome. The sensitivity and specificity of procalcitonin for TVD were $67.7 \%$ and $58.9 \%$, respectively. Procalcitonin may be a prognostic factor such as troponin, and PMR and NLR may also have an impact on prognosis.

It has been shown that Neutrophil/lymphocyte ratio may be used as a systemic inflammation marker for predicting morbidity, mortality and the risk of MI in high-risk coronary artery disease. The study evaluated admission NLR values, in-hospital and 30-day mortality in acute coronary syndrome and revealed the mortality rate as $4 \%$ in low NLR group, $10 \%$ in moderate NLR group and $19 \%$ in high NLR group at the end of 30 days $^{20}$. Tamhane et al. ${ }^{21}$ studied the admission NLR values with in-hospital and 6-month mortality in patients with ACS and found that NLR was an independent predictor of the in-hospital and 6-month mortality. Also, it was reported that the categorization of patients according to GRACE scores as low, moderate and high revealed that the patients with NLR> 4.7 had the highest mortality rates.

In our study, NLR was low in the UA group, which had low-level inflammation, whereas it was high in STEMI and NSTEMI were high. The highest NLR values were observed in high lateral MI. High rates of complications, TVD and mortality were observed in patients with NLR> 4.7 and above. The sensitivity of NLR for mortality and TVD was $89.3 \%$ and $90.2 \%$ with the specificity of $71.7 \%$ and $70.8 \%$, respectively.

Platelet activation and aggregation play an important role in the pathogenesis of ACS. Cameron et al. ${ }^{22}$ found that patients with ACS had higher platelet volumes and lower platelet counts compared to the controls. Besides, elevated MPV was associated with poor prognosis in $\mathrm{MI}^{23,24}$. Chu et al. ${ }^{25}$ found that there was a correlation between MPV and MI in their meta-analysis, but they could not determine MPV and the size of the infarct.

In our study, we found a correlation between MPV and ACS. PMR was high in the UA group, which had low-level inflammation, whereas it was low in STEMI and NSTEMI. PMR was negatively correlated with the Gensini Score, troponins, procalcitonin, and NLR while it was positively correlated with LVEF in patients with the acute coronary syndrome. High Gensini score and low ejection fraction were associated with poor prognosis and increased mortality. The sensitivity of PMR for mortality and TVD were $88.9 \%$ and $87.9 \%$ with the specificity of $81.3 \%$ and $76.1 \%$, respectively.

In this study, we found that the levels of procalcitonin, troponin, PMR and NLR were elevated in ACS. These levels were found to be low in cases with low-level inflammation, such as unstable angina, accompanied by high levels of PMR. However, NLR was significantly higher in STEMI and NSTEMI, whereas PMR was lower. Such outcomes suggest that these values are correlated with the severity of atherosclerosis in ACS and are released due to various inflammatory events occurring during MI.

\section{Study limitations}

The single-center and retrospective design of the study were among the major limitations, along with the difficulties in retrieving the data. Also, we could not obtain information about the patients' medication history, secondary factors and risk factors for coronary artery disease which could affect the prognosis. The gender and gensini score, which is a compatible study with the literature, was not used in the discussion so that the article could not be further extended. Besides, multiple test analyzes were not studied with the same idea. The inaccessibility of electrocardiography, troponin and procalcitonin data after discharge were among the major limitations of the study.

\section{Conclusion}

Elevated procalcitonin levels, along with PMR and NLR, may be used as a marker for prognosis in respect to TVD, complications, and mortality in patients with ACS, independent of Tn. We believe that it will be useful to carry out further prospective studies.

\section{References}

1. Elliot WJ. Cardiovascular risk factors. Which ones can and should be remedied? Postgrad med. 1994; 96: 49-58.

2. Kristensen SD.The platelet-vessel wall interaction in experimental atherosclerosis and ischaemic heart disease with special reference to thrombopoiesis. Dan Med Bull. 1992 Apr; 39(2): 110-27.

3. Endler G, Klimesch A, Sunder-Plassmann H, et al. Mean platelet volume is an independent risk factor for myocardial infarction but not for coronary artery disease. Br J Haematol. 2002; 117: 399-404.

4. Inhibition of the platelet glycoprotein IIb/IIIa receptor with tirofiban in uns-table angina and non-Q-wave myocardial infarction. Platelet Receptor Inhibition in Ischemic Syndrome Management in Patients Limited by Unstable Signs and Symptoms (PRISM-PLUS) Study Investigators. N Engl J Med. 1998; 338(21): 1488- 97.

5. Davies MJ, Thomas AC. Thrombosis and acute coronary artery lesions in sudden cardiac ischemic death. N Engl J Med. 1984; 310(18): 1137 40 .

6. Naruko T, Ueda M, Haze $\mathrm{K}$, et al. Neutrophil infiltration of culprit lesions in acute coronary syndromes. Circulation. 2002; 106: 2894900.

7. Balta S, Demirkol S, Celik T, et al. Association between coronary artery ectasia and neutrophil-lymphocyte ratio. Angiology. 2013; 64 (8): 627-32.

8. Panteghini M, Pagani F, Yeo KT, et al. Evaluation of imprecision for cardiac troponin assays at low-range concentrations. Clin Chem. 2004; 50: 327-32.

9. Maruna P, Nedelnıkova K, Gurlich R. Physiology and genetics of procalcitonin. Physiol Res. 2000; 49(1): 57-61. 
10. Whicher J, Bienvenu J, Monneret G. Procalcitonin as an acute phase marker. Ann Clin Biochem. 2001; 38: 483- 93.

11. Ataoglu HE, Yilmaz F, Uzunhasan I, et al. Procalcitonin: A novel cardiac marker with prognostic value in acute coronary syndrome. J Int Med Res. 2010; 38: 52-61.

12. 12. Kelly D, Khan SQ, Dhillon O, et al. Procalcitonin as a prognostic marker in patients with acute myocardial infarction. Biomarkers. 2010; 15: 325 -31.

13. AHA/ACC Guideline for the Management of Patients With Non-STElevation Acute Coronary Syndromes. Circulation. 2014; 130: 344426.

14. Gensini GG. A more meaningful scoring system for determining the severity of coronary heart disease. Am J Cardiol. 1983; 51: 606.

15. Biasucci LM, Biasillo G, Stefanelli A. Procalcitonin and acute coronary syndromes: a new biomarker for an old disease. Intern Emerg Med. 2009; 4: 363-5.

16. Sinning CR, Sinning JM, Schulz A, et al; AtheroGene Study Investigators. Association of serum procalcitonin with cardiovascular prognosis in coronary artery disease. Circ J. 2011; 75: 1184-91.

17. Antman EM, Tansijevic MJ, Thompson B, et al. Cardiac-specific troponin I levels to predict the risk of mortality in patients with acute coronary syndromes. New Engl J Med. 1996; 335: 1342-9.
18. Kafkas N, Venetsanou K, Patsilinakos S, et al. Procalcitonin in acute myocardial infarction. Acute Card Care. 2008; 10: 30-36.

19. Senturk T, Cordan J, Baran I, et al. Procalcitonin in patients with acute coronary syndrome: Correlation with high-sensitive C-reactive protein, prognosis, and severity of coronary artery disease. Acta Cardiol. 2007; 62: 135-41.

20. Muhammed Suliman MA, Bahnacy Juma AA, Ali Almadhani AA, et al. Predictive value of neutrophil to lymphocyte ratio in outcomes of patients with the acute coronary syndrome. Arch Med Res. 2010; 41: 618-22.

21. Tamhane UU, Aneja S, Montgomery D, et al. Association between admission neutrophil to lymphocyte ratio and outcomes in patients with the acute coronary syndrome. Am J Cardiol. 2008; 102: 653-7.

22. Cameron HA, Phillips R, Ibbotson RM, et al. Platelet size in myocardial infarction. Br Med J. 1983; 287: 449-451.

23. Martin JF, Bath PM, Burr ML. Influence of platelet size on outcome after myocardial infarction. Lancet. 1991; 338: 1409-11.

24. Pabón OP, Nieto BF, Moríñigo MJL, et al. The effect of the mean platelet volume on the short-term prognosis of the acute myocardial infarct. Rev Esp Cardiol. 1998; 51: 816-22.

25. Chu SG, Becker RC, Berger PB, et al. Mean platelet volume as a predictor of cardiovascular risk: a systematic review and metaanalysis. J Thromb Haemost. 2010; 8: 148-56. 